\title{
Studies on the Radiological Impact of Oil and Gas Activities in Oil Mineral Lease 30 (Oml30) Oil Fields in Delta State, Nigeria
}

\section{GO Avwiri ${ }^{1}$ and EO Agbalagba ${ }^{2 *}$}

${ }^{1}$ Department of Physics, University of Port Harcourt, Nigeria

${ }^{2}$ Department of Physics, Federal University of Petroleum Resources, Effurun, Nigeria

\begin{abstract}
A preliminary studies on the radiological impact of oil and gas exploration activities in the oil and gas production land area of Delta State has been carried out insitu, using two radiation meters (Digilert 50 and 100) synchronized and calibrated and a geographical positioning system (GPS). Readings were taken in nine different facilities in each of the oil and gas fields and one sample each in their host communities. Measured radiation values in the oil field facilities ranged from $0.011 \pm 0.003 \mathrm{mRh}^{-1}$ in Evwreni campsite to $0.031 \pm 0.01 \mathrm{mRh}^{-1}$ at the Otorogu gas plant. Mean field exposure rates/equivalent dose rates in the oil fields ranged from $0.016 \pm 0.006 \mathrm{mRh}^{-1}\left(1.4 \pm 0.5 \mathrm{mSvy}^{-1}\right)$ to $0.0213 \pm 0.008 \mathrm{mRh}^{-1}\left(1.8 \pm 0.7 \mathrm{mSvy}^{-1}\right)$. While in the host communities values ranged from $0.011 \pm 0.003 \mathrm{mRh}^{-1}(0.9$ $\left.\pm 0.3 \mathrm{mSvy}^{-1}\right)$ in Evwreni community to $0.021 \pm 0.007 \mathrm{mRh}^{-1}\left(1.8 \pm 0.7 \mathrm{mSvy}^{-1}\right)$ in Otujeremi town and the control study area value been $0.009 \pm 0.002 \mathrm{mRh}^{-1}\left(0.8 \pm 0.06 \mathrm{mSvy}^{-1}\right)$. The results show that all the oil and gas fields and host communities except Evwreni community yearly radiation dose rate exceeded the $1 \mathrm{mSvy}^{-1}$ maximum permissible limit recommend for the public and non-nuclear industrial environment by International Council on Radiological Protection [1]. All the oil fields and host community except Ughelli East and Evwreni community radiation levels exceeded the normal world average BIR level of $0.013 \mathrm{mRh}^{-1}$ and other reported values in similar environment. This shows that the oil fields environment and the host communities have been impacted radiologically. However, these results obtained may not have immediate health hazard, but will pose some long-term health side effects on the staff working in the facilities and residents of the host communities. Interim proactive measures are recommended while further and a detail study is ongoing.
\end{abstract}

Keywords: Radiological impact; Oil and Gas facilities; OML30; Delta state

\section{Introduction}

Radiation plays an important and sometimes vital role in our everyday lives. Every day each of us is exposed to naturally occurring quantities of radiation. We are exposed to these radioactive materials through the air we breathe, the soil on which we walk, the water we drink, the food we eat and even within our bodies [2]. Monitoring for radioactive materials is of primary importance for environmental protection, but rapid and accurate methods for the assay of radioactivity are essential [3].

Crude oil and other petroleum related products is a naturally occurring liquid mineral deposited beneath the earth surface. Its occurrence is sometimes accompanied with the existence of natural gas. The oil, gas and associated gas are contaminated generally with radionuclide in the earth crust and drilling material. All these provide the source of radiation such as $\alpha, \beta$ and $\gamma$ radiation often found in the petroleum matrix [4].

Gamma rays are known to be highly penetrating and are part products of the radioactive materials containing radon that may be ingested or inhaled into the human body, during repairs and maintenance of oil facilities. If inhaled the dust particles and aerosols containing radon may attach themselves to the lungs where gamma rays emitted in the decay may pose increase risk of lung cancer, eye cataracts and mental imbalances to personnel and host communities [4].

In recent time, researchers have found a strong correlation between radiation exposure and health hazard on workers in this environment eco-system [5] which are attributed to the industries input raw materials, effluents discharged as in gas flare and output products.
Elena and Gracea [6] conduct environmental monitoring of radioactivity in the surroundings of six oil fields in Bacau and Braila districts and reported that from radiological point of view, the situation does not pose any immediate concern. However, the high radium-226 content of oil field formation waters could lead to environmental pollution. Laogun et al. [4] studied the variation in well-Heads gamma radiation levels at an oil field in Ologbo, Edo state and reported that the values obtained are fairly higher than the normal background level but are in agreement with the International Atomic Energy Agency's standard on background ionizing radiation level for such environment. Also, the Rail Road Commission of Texas [7] reported that naturally occurring radioactive materials (NORMS) associated with oil and gas production originated in subsurface and contain radioactive materials like Uranium and Thorium and their daughter progenies (Ra-226 and Ra-228).

Avwiri et al. [5] studied the terrestrial radiation around oil and gas facilities in Ughelli region of Nigeria and reported an average value range of $12.00 \pm 0.10 \mu \mathrm{Rh}^{-1}(5.33 \pm 0.35 \mu \mathrm{Sv} / \mathrm{wk})$ to $22.00 \pm 2.1 \mu \mathrm{Rh}$ ${ }^{1}(9.79 \pm 0.16 \mu \mathrm{Sv} / \mathrm{wk})$ in the oil fields and $09.00 \pm 1.0 \mu \mathrm{Rh}^{-1}$ to 11.00

*Corresponding author: EO Agbalagba, Department of Physics, Federal University of Petroleum Resources, Effurun, Nigeria, Tel: +2348037434510 E-mail: ezek64@yahoo.com

Received October 20, 2011; Accepted February 06, 2012; Published February 08, 2012

Citation: Avwiri GO, Agbalagba EO (2012) Studies on the Radiological Impact of Oil and Gas Activities in Oil Mineral Lease $30(\mathrm{Oml} 30)$ Oil Fields in Delta State, Nigeria. J Pet Environ Biotechnol 3:115. doi:10.4172/2157-7463.1000115

Copyright: $\odot 2012$ Avwiri GO, et al. This is an open-access article distributed under the terms of the Creative Commons Attribution License, which permits unrestricted use, distribution, and reproduction in any medium, provided the original author and source are credited. 
$\pm 0.5 \mu \mathrm{Rh}^{-1}$ in the host communities. They concluded that though the radiation values are within international standard and are in consonant with other reported values in the country, the BIR levels exceeded the normal background level.

EPA [8] on environmental, health and safety online, stated that the more radiation dose from oil and gas installation on a person or worker receives, the greater the chance of developing cancer, leukemia, eye cataracts, erythema, hematological depression and incidence of chromosome aberrations. This may not appear until many years after the radiation dose is received (typically, 10-40 years).

Through temperature and pressure changes that occur in the course of oil and gas production operations, radium-226 and radium-228 found in produced waters may co-precipitate with barium sulfate scale in well tubules and surface equipment. Concentrations of radium-226 and radium-228 may also occur in sludge that accumulates in oil field pits and tanks. These solids become sources of hydrocarbon NORM waste [9]. In gas processing activities, NORM generally occurs as radon gas in the natural gas stream. Radon decay elements occur as a film on the inner surface of inlet lines, treating units, pumps and valves principally associated with propylene, ethane and propane processing streams. Production and processing equipment may contain residual quantities of NORM- contaminated water, scale or sludge that can cause disposal and exposure problems when the equipment is takenoff line for maintenance, repair or replacement. Workers employed in the area of cutting and reaming oil field pipe, removing solids from tanks, pits and refurbishing gas processing equipment may be exposed to particles containing levels of alpha and or beta emitting radionuclide that could pose health risks if inhaled or ingested [10]

The objective of this study is therefore to assess the radiological impact on the environment and population of the oil/gas industry that is non-nuclear industry. This study will also give precise and accurate information on the background ionizing radiation (BIR) levels of these flow stations and their host communities in the Niger Delta State and add to the data on background radiation levels in oil facilities in the region. The health implications of the obtained results on the fields' workers and residents of the host communities were examined.

\section{Experimental Method}

The studied oil fields are within the Oil Mining Lease 30 (OML 30) of the production land area of Niger Delta Shown in figure 1. An insitu approach of the background radiation levels measurement was preferred to enable samples maintain their original environmental characteristics. Two radiation meters, Digilert 50 and 100 nuclear radiation monitors (S.E International Inc. Summer Town, USA), which contain a Geiger Muller tube, each capable of detecting; $\alpha, \beta, \gamma$ and $\mathrm{x}$-rays within the temperature range of -10 to $50^{\circ} \mathrm{C}$ were used.

Prior to use, the two meters were synchronize by resetting them. Readings were taken on each operational scale at the time of the calibrations with check sources and repeated every 5 minutes. The standard errors detected were $\pm 8 \%$ and $\pm 5 \%$ for digilert 50 and 100 respectively. During field measurements, the tube of the radiation meters were held at a standard height of $1.0 \mathrm{~m}$ above the ground and placed at about $2.0 \mathrm{~m}$ away from the facilities. The windows of the radiation meters were first oriented vertically downward and then toward the oil/gas facility $[4,5]$. The geographical positioning system (GPS) reading for the particular facility location was recorded.

For optimum meter responds and results, measurements were carried out between 1300 to 1600 hours each day, since the radiation meters have maximum response to environmental radiation within these hours [11]. At each facility, three readings were obtained simultaneously at 300 secs each facility and their average values computed. In a field, nine different facilities radiation levels were taken to ensure adequate coverage of the oil field facilities. Also one radiation level measurement was obtained outside in each oil field host communities.

To estimate the whole body equivalent dose rate, the researcher used the National Council of Radiation Protection and Measurements (NCRP, 1993) recommendation which stipulates that

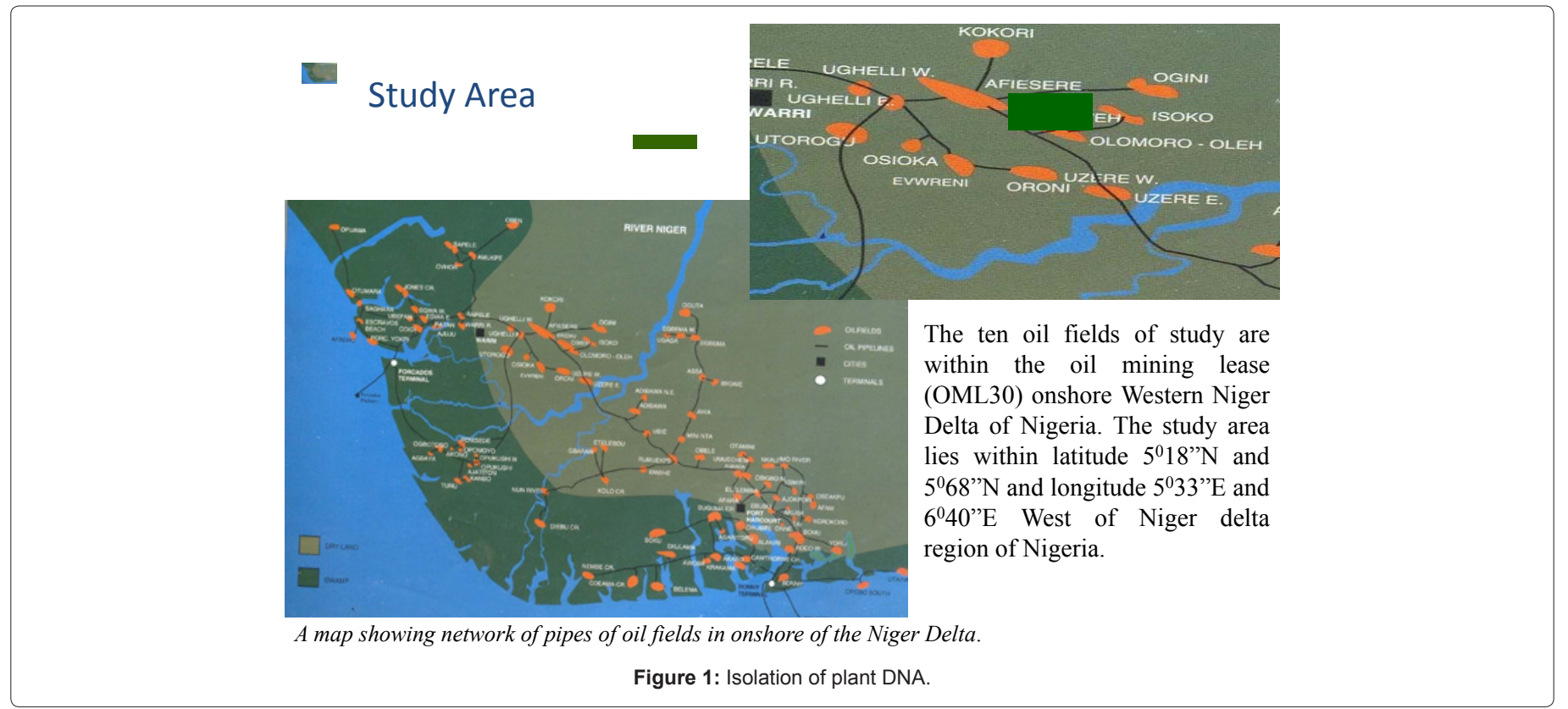


Citation: Avwiri GO, Agbalagba EO (2012) Studies on the Radiological Impact of Oil and Gas Activities in Oil Mineral Lease 30 (Oml30) Oil Fields in Delta State, Nigeria. J Pet Environ Biotechnol 3:115. doi:10.4172/2157-7463.1000115

Page 3 of 8

$$
1 m R h^{-1}=(\text { üüüüx } \quad x / / 100) m S v y^{-1}
$$

\section{Results and Discussion}

Tables 1-10 show the results of the in situ measurement carried out in the nine studied oil and gas fields and one host community. The facilities exposure rate in the oil fields ranged from $0.011 \pm 0.03 \mathrm{mRh}$ ${ }^{1}$ at the campsite in Evwreni oil fields to $0.031 \pm 0.010 \mathrm{mRh}^{-1}$ at the Otorogu gas plant in Otorogu oil and gas field. The high values obtained at the Otorogu gas plant may be attributed to the high concentration of randon accompanying natural gas, which is in abundance in this environment.

The mean exposure rates/equivalent dose rates in the oil fields ranged from $0.016 \pm 0.006 \mathrm{mRh}^{-1}\left(1.350 \pm 0.50 \mathrm{mSvy}^{-1}\right)$ in Evwreni field to $0.021 \pm 0.008 \mathrm{mRh}^{-1}\left(1.79 \pm 0.70 \mathrm{mSvy}^{-1}\right)$ in Otorogu oil and gas field (Figure 1). The low level obtained at Evwreni oil and gas field could be attributed to the shutdown in operations at the oil field. While the high radiation level recorded at some of the oil and gas field especially at Otorogu field may be as a result of the ongoing development of new oil wells and the turnaround maintenance going on in some major facilities within these oil and gas fields. The mean field radiation values obtained from the oil and gas field show that, Ughelli East, Kokori, Eriemu, Evwreni, Oweh, Olomoro-Oleh oil and gas fields are still within the $1.0 \mathrm{mSvy}^{-1}$ maximum permissible limit for non-nuclear work environment and the general public, recommended by European Council for Nuclear Research [12] and International Council on

\begin{tabular}{|c|c|c|c|c|c|c|}
\hline \multirow{2}{*}{$\mathrm{S} / \mathrm{N}$} & \multirow{2}{*}{ SAMPLED AREA } & \multirow{2}{*}{ GEOGRAPHICAL LOCATION } & \multicolumn{2}{|c|}{ RADIATION LEVEL $\quad \mathrm{mRh}^{-1}$} & \multirow{2}{*}{ AVE. RAD. VALUE } & \multirow{2}{*}{$\begin{array}{c}\text { EQ. DOSE } \\
\text { mSvy }^{-1}\end{array}$} \\
\hline & & & RAD 50 & RAD 100 & & \\
\hline 1 & Crude Flow Pipe & NO5 32.297' E005 53.780' & 0.019 & 0.018 & $0.019 \pm 0.004$ & $1.6 \pm 0.3$ \\
\hline 2 & Natural Gas Compressor & NO5 26.021' E005 52.940 & $0.025^{\prime}$ & 0.019 & $0.022 \pm 0.008$ & $1.9 \pm 0.7$ \\
\hline 3 & Flow station entrance & NO5 26..057’ E005 52.926' & 0.017 & 0.018 & $0.018 \pm 0.007$ & $1.5 \pm 0.6$ \\
\hline 4 & Well 7 & NO5 25.918' E005 53.014' & 0.021 & 0.024 & $0.223 \pm 0.009$ & $1.9 \pm 0.8$ \\
\hline 5 & Pegging Manifold & N05 26.062' E005 52.901"' & 0.019 & 0.021 & $0.020 \pm 0.008$ & $1.7 \pm 0.6$ \\
\hline 6 & Well 10 & N05 25.671' E005 52.930' & 0.016 & 0.018 & $0.017 \pm 0.006$ & $1.4 \pm 0.5$ \\
\hline 7 & Flare Stack Site & N05 26.141 E005 52.653 & 0.024 & 0.025 & $0.025 \pm 0.011$ & $2.1 \pm 0.8$ \\
\hline 8 & Well 5 & NO5 25.701' E005 52.608' & 0.018 & 0.020 & $0.019 \pm 0.009$ & $1.6 \pm 0.7$ \\
\hline 9 & Olorogu Gas Plant & NO5 25.701' E005 52.608' & 0.028 & 0.034 & $0.031 \pm 0.010$ & $2.6 \pm 0.8$ \\
\hline \multirow[t]{2}{*}{10} & Otujeremi Town & NO5 25.865' E005 52.567' & 0.022 & 0.020 & $0.021 \pm 0.007$ & $1.8 \pm 0.6$ \\
\hline & \multicolumn{2}{|l|}{ MEAN FIELD LEVELS } & & & $0.021 \pm 0.008$ & $1.8 \pm 0.7$ \\
\hline
\end{tabular}

Table 1: Otorogu Oil and Gas Field Background Radiation Level.

\begin{tabular}{|c|c|c|c|c|c|c|}
\hline \multirow{2}{*}{$S / N$} & \multirow{2}{*}{ SAMPLED AREA } & \multirow{2}{*}{ GEOGRAPHICAL LOCATION } & \multicolumn{2}{|c|}{ RADIATION LEVEL $\mathrm{mRh}^{-1}$} & \multirow{2}{*}{$\begin{array}{l}\text { AVE. RAD. VALUE } \\
\mathrm{mRh}^{-1}\end{array}$} & \multirow{2}{*}{$\begin{array}{l}\text { EQ. DOSE } \\
\text { mSvy }^{-1}\end{array}$} \\
\hline & & & RAD 50 & RAD 100 & & \\
\hline 1 & Crude Flow Pipe & NO5 32.297' E005 53.780' & 0.019 & 0.018 & $0.019 \pm 0.003$ & $1.6 \pm 0.3$ \\
\hline 2. & Flow station entrance & NO5 32..303' E005 53.782' & 0.029 & 0.024 & $0.027 \pm 0.008$ & $2.2 \pm 0.7$ \\
\hline 3 & Well 7 & NO5 32.338' E005 53.7954' & 0.024 & 0.018 & $0.021 \pm 0.006$ & $1.8 \pm 0.5$ \\
\hline 4 & Limer \& Serier (L\&S) Tango piple & N05 32.279' E005 53.771' & 0.023 & 0.020 & $0.022 \pm 0.006$ & $1.9 \pm 0.5$ \\
\hline 5 & Crude oil control & N05 32.275' E005 53.759 & 0.016 & 0.018 & $0.017 \pm 0.005$ & $1.4 \pm 0.4$ \\
\hline 6 & Flare knockout vessel & N05 32.282' E005 53.720' & 0.015 & 0.018 & $0.017 \pm 0.006$ & $1.4 \pm 0.5$ \\
\hline 7 & Flare control valve & N05 32.295' E005 53.685 & 0.018 & 0.014 & $0.016 \pm 0.005$ & $1.4 \pm 0.4$ \\
\hline 8 & Flare stack point & N05 32.307' E005 53.678 & 0.016 & 0.015 & $0.016 \pm 0.005$ & $1.3 \pm 0.4$ \\
\hline 9 & Well 2 & N05 32.112' E005 53.802' & 0.018 & 0.020 & $0.019 \pm 0.007$ & $1.6 \pm 0.6$ \\
\hline \multirow[t]{2}{*}{10} & Ekakpamre community & N05 31.071 E005 54.170 & 0.021 & 0.017 & $0.019 \pm 0.008$ & $1.6 \pm 0.7$ \\
\hline & MEAN FIELD & & & & $0.019 \pm 0.005$ & $1.6 \pm 0.4$ \\
\hline
\end{tabular}

Table 2: Ughelli West Oil and Gas Field Background Radiation Level.

\begin{tabular}{|c|c|c|c|c|c|c|}
\hline \multirow{2}{*}{$\mathrm{S} / \mathrm{N}$} & \multirow{2}{*}{ SAMPLED AREA } & \multirow{2}{*}{ GEOGRAPHICAL LOCATION } & \multicolumn{2}{|c|}{ RADIATION LEVEL mRh ${ }^{-1}$} & \multirow{2}{*}{ AVE. RAD. VALUE $\mathrm{mRh}^{-1}$} & \multirow{2}{*}{$\begin{array}{l}\text { EQ. DOSE } \\
\text { mSvy }^{-1}\end{array}$} \\
\hline & & & RAD 50 & RAD 100 & & \\
\hline 1 & Flow station entrance & NO5 30.850' E005 56.233' & 0.021 & 0.019 & $0.020 \pm 0.008$ & $1.7 \pm 0.7$ \\
\hline 2. & Control station (UNC) & NO5 30.856' E005 56.229' & 0.019 & 0.015 & $0.017 \pm 0.006$ & $1.4 \pm 0.5$ \\
\hline 3 & L \& S tango crude pipe & NO5 30.860' E005 56.210' & 0.018 & 0.018 & $0.018 \pm 0.007$ & $1.5 \pm 0.6$ \\
\hline 4 & Crude and Gas control valve & N05 30.864' E005 56.117 & 0.015 & 0.017 & $0.016 \pm 0.005$ & $1.3 \pm 0.4$ \\
\hline 5 & UPS Manifold & N05 30.750' E005 56.272 & 0.017 & 0.020 & $0.019 \pm 0.007$ & $1.6 \pm 0.6$ \\
\hline 6 & Flare site & N05 30.984' E005 56.271' & 0.016 & 0.04 & $0.015 \pm 0.005$ & $1.3 \pm 0.4$ \\
\hline 7 & Ughelli East Buster station & N05 31.004' E005 55.910' & 0.018 & 0.018 & $0.018 \pm 0.004$ & $1.5 \pm 0.3$ \\
\hline 8 & Well 5 & N05 30.783’ E005 56.310 & 0.016 & 0.014 & $0.015 \pm 0.007$ & $1.3 \pm 0.6$ \\
\hline \multirow[t]{3}{*}{9} & NGC station & N05 30.860' E005 56.199' & 0.019 & 0.020 & $0.019 \pm 0.008$ & $1.6 \pm 0.7$ \\
\hline & Eruemukohwara community & N05 31.598' E005 56.409' & 0.011 & 0.014 & $0.012 \pm 0.004$ & $1.1 \pm 0.3$ \\
\hline & \multicolumn{2}{|l|}{ MEAN FIELD LEVEL } & & & $0.017 \pm 0.007$ & $1.5 \pm 0.6$ \\
\hline
\end{tabular}

Table 3: Ughelli East Oil and Gas Field Background Radiation Level. 
Citation: Avwiri GO, Agbalagba EO (2012) Studies on the Radiological Impact of Oil and Gas Activities in Oil Mineral Lease 30 (Oml30) Oil Fields in Delta State, Nigeria. J Pet Environ Biotechnol 3:115. doi:10.4172/2157-7463.1000115

\begin{tabular}{|c|c|c|c|c|c|c|}
\hline \multirow{2}{*}{$\mathbf{S} / \mathbf{N}$} & \multirow{2}{*}{ SAMPLED AREA } & \multirow{2}{*}{ GEOGRAPHICAL LOCATION } & \multicolumn{2}{|c|}{ RADIATION LEVEL mRh ${ }^{-1}$} & \multirow{2}{*}{$\begin{array}{l}\text { AVE. RAD. VALUE } \\
\mathrm{mRh}^{-1}\end{array}$} & \multirow{2}{*}{$\begin{array}{l}\text { EQ. DOSE } \\
\text { mSvy }^{-1}\end{array}$} \\
\hline & & & RAD 50 & RAD 100 & & \\
\hline 1 & Flow and compressor station gate & NO5' 32.888' E006 00.898' & 0.017 & 0.020 & $0.019 \pm 0.008$ & $1.6 \pm 0.7$ \\
\hline 2. & Manifold & NO5' 3 2.871' E006' 00.892' & 0.030 & 0.028 & $0.029 \pm 0.009$ & $2.4 \pm 0.7$ \\
\hline 3 & Flare control valve & NO5' 32.899' E006'00.808' & 0.018 & 0.020 & $0.019 \pm 0.007$ & $1.6 \pm 0.6$ \\
\hline 4 & L \& S Tango flow crude pipe & N05' 32.903' E005 56.117 & 0.014 & 0.015 & $0.015 \pm 0.007$ & $1.2 \pm 0.5$ \\
\hline 5 & Natural gas compressor (NGC) station & N05' 32.652' E006. 01.138 & 0.021 & 0.019 & $0.021 \pm 0.008$ & $1.7 \pm 0.7$ \\
\hline 6 & Flare knockout vessel & N05' 32.801' E006 00.776' & 0.016 & 0.015 & $0.016 \pm 0.006$ & $1.3 \pm 0.5$ \\
\hline 7 & Flare site & N05 32.906' E005 00.801' & 0.023 & 0.020 & $0.022 \pm 0.009$ & $1.8 \pm 0.8$ \\
\hline 8 & Well 27 & N05” 32.863' E005 00.982 & 0.018 & 0.017 & $0.018 \pm 0.008$ & $1.5 \pm 0.6$ \\
\hline 9 & Well 13 & N05" 32.783' E005 01.035' & 0.022 & 0.025 & $0.024 \pm 0.008$ & $2.0 \pm 0.7$ \\
\hline \multirow[t]{2}{*}{10} & Emeragha community & N05 32.582' E005 01.530' & 0.019 & 0.018 & $0.019 \pm 0.006$ & $1.6 \pm 0.5$ \\
\hline & \multicolumn{2}{|l|}{ MEAN FIELD LEVEL } & & & $0.020 \pm 0.008$ & $1.7 \pm 0.6$ \\
\hline
\end{tabular}

Table 4: Afiesere Oil and Gas Field Background Radiation Level.

\begin{tabular}{|c|c|c|c|c|c|c|}
\hline \multirow{2}{*}{$\mathbf{S} / \mathbf{N}$} & \multirow{2}{*}{ SAMPLED AREA } & \multirow{2}{*}{ GEOGRAPHICAL LOCATION } & \multicolumn{2}{|c|}{ RADIATION LEVEL mRh-1 } & \multirow{2}{*}{$\begin{array}{l}\text { AVE. RAD VALUE } \\
\mathrm{mRh}^{-1}\end{array}$} & \multirow{2}{*}{$\begin{array}{l}\text { EQ. DOSE } \\
\mathrm{mSvy}^{-1}\end{array}$} \\
\hline & & & RAD 50 & RAD 100 & & \\
\hline 1 & Manifold & NO5' 38.624' E006 04.321' & 0.017 & 0.025 & $0.021 \pm 0.008$ & $1.8 \pm 0.7$ \\
\hline 2. & Flow station Gate & NO5' 38.641' E006' 04'224' & 0.018 & 0.020 & $0.019 \pm 0.009$ & $1.06 \pm 0.8$ \\
\hline 3 & Natural Gas compressor (NGC) station & NO5' 38.638' E006' 04.215' & 0.018 & 0.022 & $0.021 \pm 0.010$ & $1.7 \pm 0.8$ \\
\hline 4 & L \& S Tangle flow crude pipe & N05' 38.601' E006 04.226' & 0.016 & 0.014 & $0.015 \pm 0.007$ & $1.3 \pm 0.6$ \\
\hline 5 & Control valve (crude) & N05' 39.012' E006. 04.171 & 0.018 & 0.020 & $0.019 \pm 0.007$ & $1.6 \pm 0.6$ \\
\hline 6 & Flare knockout drum & N05'39.016' E006 0466' & 0.017 & 0.020 & $0.019 \pm 0.006$ & $1.6 \pm 0.5$ \\
\hline 7 & Flare stock site & N05' 39.108' E005 00.801' & 0.017 & 0.015 & $0.016 \pm 0.006$ & $1.4 \pm 0.5$ \\
\hline 8 & Well 13, 34 \& 35 & N05" 38.844' E006" 04.030' & 0.020 & 0.023 & $0.022 \pm 0.011$ & $1.8 \pm 0.9$ \\
\hline 9 & Flare control valve & N05" 39.112' E006 04.192' & 0.016 & 0.014 & $0.015 \pm 0.008$ & $1.3 \pm 0.7$ \\
\hline \multirow[t]{2}{*}{10} & Erhioke Community & N05 38.602' E006" 04.227' & 0.014 & 0.013 & $0.014 \pm 0.004$ & $1.2 \pm 0.3$ \\
\hline & \multicolumn{2}{|l|}{ MEAN FIELD LEVEL } & & & $0.018 \pm 0.007$ & 1.50 .6 \\
\hline
\end{tabular}

Table 5: Kokori Oil and Gas Field Background Radiation Level.

\begin{tabular}{|c|c|c|c|c|c|c|}
\hline \multirow{2}{*}{$\mathbf{S} / \mathbf{N}$} & \multirow{2}{*}{ SAMPLED AREA } & \multirow{2}{*}{ GEOGRAPHICAL LOCATION } & \multicolumn{2}{|c|}{ RADIATION LEVEL mRh-1 } & \multirow{2}{*}{$\begin{array}{l}\text { AVE. RAD. VALUE } \\
\mathrm{mRh}^{-1}\end{array}$} & \multirow{2}{*}{$\begin{array}{l}\text { EQ. DOSE } \\
\text { mSvy }^{-1}\end{array}$} \\
\hline & & & RAD 50 & RAD 100 & & \\
\hline 1 & Field logistic base (FCB) & NO5' 32.770' E006 02.716' & 0.022 & 0.016 & $0.019 \pm 0.009$ & $1.6 \pm 0.8$ \\
\hline 2. & Well 3 & NO5' 31.264' E006' 03501 & 0.014 & 0.019 & $0.017 \pm 0.007$ & $1.4 \pm 0.6$ \\
\hline 3 & Pegging manifold & NO5' 31.550' E006' 03.430' & 0.016 & 0.013 & $0.015 \pm 0.004$ & $1.2 \pm 0.3$ \\
\hline 4 & N.G.C Station & N05' 31.211' E006 03.428' & 0.019 & 0.017 & $0.018 \pm 0.008$ & $1.5 \pm 0.7$ \\
\hline 5 & Flow station Gate & N05' 31.218' E006. 03.488' & 0.012 & 0.014 & $0.013 \pm 0.005$ & $1.1 \pm 0.4$ \\
\hline 6 & Gas Vent (knockout drum) & N05' 31.488' E006 03.498' & 0.017 & 0.018 & $0.015 \pm 0.007$ & $1.3 \pm 0.6$ \\
\hline 7 & Flare stack site & N05' 31.305' E006 03.519' & 0.013 & 0.019 & $0.016 \pm 0.006$ & $1.4 \pm 0.5$ \\
\hline 8 & L \& S Tango Crude flow pipe & N05" 31.246' E006" 03.473' & 0.013 & 0.016 & $0.015 \pm 0.005$ & $1.2 \pm 0.4$ \\
\hline 9 & Well $13 \& 19$ & N05” 32 .181' E006' 02.251' & 0.018 & 0.020 & $0.019 \pm 0.007$ & $1.6 \pm 0.6$ \\
\hline \multirow[t]{2}{*}{10} & Gana Agbarh-otor community & N05 38.578' E006" 03.75' & 0.017 & 0.014 & $0.016 \pm 0.007$ & $1.3 \pm 0.6$ \\
\hline & \multicolumn{2}{|l|}{ MEAN FIELD LEVEL } & & & $0.016 \pm 0.006$ & $1.4 \pm 0.5$ \\
\hline
\end{tabular}

Table 6: Eriemu Oil and Gas Field Background Radiation Level.

Radiological Protection [1]. However, Otorogu, Ughelli West, Afiesere and Uzere West and East oil and gas fields exceeded this maximum value of $1.0 \mathrm{mSvy}^{-1}$ [1]. This calls for a concern and detailed studies of the oil fields to ascertain the level of radiological impact of these fields' workers, host communities and the immediate environment.

The exposure rates obtained in the host communities ranged from $0.011 \pm 0.003 \mathrm{mRh}^{-1}\left(0.925 \pm 0.300 \mathrm{mSvy}^{-1}\right)$ in Evwreni community to $0.021 \pm 0.007 \mathrm{mRh}^{-1}\left(1.770 \pm 0.670 \mathrm{mSvy}^{-1}\right)$ in Otujeremi town. It was generally observed that the closer the facilities to the host community the higher the impact level, thus proximity plays an important role in the radiation impact and distribution. These results obtained in the host communities show that all communities equivalent dose rate exceeded the [1] $1.0 \mathrm{mSvy}^{-1}$ maximum (Table 11) permissible limit recommended, for the public, which may result in some health hazard in this environment. Shows the comparison of the studied oil fields and host communities radiation data. The percentage deviation is least at Ughelli West oil and gas field with a percentage difference of 0.30 and maximum at Ughelli East oil and gas field with a percentage difference of 39.10. This could also be attributed to the proximity of the oil and gas facilities to the host communities. The result obtained from the control site (a non-oil bearing community with the same geological, hydrological and geomorphologic features with studied fields) is 0.009 $\pm 0.002 \mathrm{mRh}^{-1}\left(0.756 \pm 0.17 \mathrm{mSvy}^{-1}\right)$, which show a great difference with the host communities BIR levels and dose value is below the [1] 1.0 $\mathrm{mSvy}^{-1}$ maximum permissible limit recommended, for the public. 
Citation: Avwiri GO, Agbalagba EO (2012) Studies on the Radiological Impact of Oil and Gas Activities in Oil Mineral Lease 30 (Oml30) Oil Fields in Delta State, Nigeria. J Pet Environ Biotechnol 3:115. doi:10.4172/2157-7463.1000115

Page 5 of 8

\begin{tabular}{|c|c|c|c|c|c|c|}
\hline \multirow{2}{*}{$\mathrm{S} / \mathrm{N}$} & \multirow{2}{*}{ SAMPLED AREA } & \multirow{2}{*}{ GEOGRAPHICAL LOCATION } & \multicolumn{2}{|c|}{ RADIATION LEVEL $\mathrm{mRh}^{-1}$} & \multirow{2}{*}{$\begin{array}{l}\text { AVE. RAD. VALUE } \\
\mathrm{mRh}^{-1}\end{array}$} & \multirow{2}{*}{$\begin{array}{l}\text { EQ. DOSE } \\
\text { mSvy }^{-1}\end{array}$} \\
\hline & & & RAD 50 & RAD 100 & & \\
\hline 1 & Camp site & NO5' 22.720' E006 02.962' & 0.011 & 0.011 & $0.011 \pm 0.003$ & $0.9 \pm 0.2$ \\
\hline 2. & Well 13 & NO5' 22.615' E006' 02 640' & 0.015 & 0.014 & $0.015 \pm 0.005$ & $1.2 \pm 0.4$ \\
\hline 3 & Manifold & NO5' 22.405' E006' 02.405' & 0.019 & 0.013 & $0.016 \pm 0.006$ & $1.4 \pm 0.5$ \\
\hline 4 & Well 1 & N05' 22.327' E006' 02.410' & 0.017 & 0.014 & $0.016 \pm 0.005$ & $1.3 \pm 0.4$ \\
\hline 5 & Flow station Gate & N05' 22.445' E006. 02.470' & 0.015 & 0.016 & $0.016 \pm 0.006$ & $1.3 \pm 0.5$ \\
\hline 6 & L \& S Tanga crude flow pipe & N05' 22.428' E006” 02 500' & 0.015 & 0.014 & $0.015 \pm 0.005$ & $1.2 \pm 0.4$ \\
\hline 7 & Gas vent (knockout drum) & N05' 22.432' E006 22.482' & 0.020 & 0.022 & $0.021 \pm 0.009$ & $1.8 \pm 0.8$ \\
\hline 8 & Flare stock site & N05" 22.361' E006" 02.451' & 0.021 & 0.018 & $0.020 \pm 0.008$ & $1.6 \pm 0.7$ \\
\hline 9 & Well 11 & N05” 22 .394' E006' 02.439' & 0.014 & 0.014 & $0.014 \pm 0.005$ & $1.2 \pm 0.4$ \\
\hline \multirow[t]{2}{*}{10} & Evwreni Community & N05' 24.243' E006" 03.451' & 0.017 & 0.014 & $0.011 \pm 0.003$ & $0.9 \pm 0.3$ \\
\hline & \multicolumn{2}{|l|}{ MEAN FIELD LEVEL } & & & $0.016 \pm 0.006$ & $1.4 \pm 0.5$ \\
\hline
\end{tabular}

Table 7: Evwreni Oil and Gas Field Background Radiation Level.

\begin{tabular}{|c|c|c|c|c|c|c|}
\hline \multirow{2}{*}{$\mathbf{S} / \mathbf{N}$} & \multirow{2}{*}{ SAMPLED AREA } & \multirow{2}{*}{ GEOGRAPHICAL LOCATION } & \multicolumn{2}{|c|}{ RADIATION LEVEL $\mathrm{mRh}^{-1}$} & \multirow{2}{*}{ AVE. RAD. VALUE $\mathrm{mRh}^{-1}$} & \multirow{2}{*}{$\begin{array}{l}\text { EQ. DOSE } \\
\text { mSvy }^{-1}\end{array}$} \\
\hline & & & RAD 50 & RAD 100 & & \\
\hline 1 & Flow Station Gate & NO5' 29.271' E006 08.101' & 0.016 & 0.012 & $0.014 \pm 0.005$ & $1.2 \pm 0.4$ \\
\hline 2. & Crude oil control valve & NO5' 08.101' E006' 08' & 0.019 & 0.019 & $0.019 \pm 0.007$ & $1.6 \pm 0.6$ \\
\hline 3 & Gas vent (knockout drum) & NO5' 29.289' E006' 08.201' & 0.017 & 0.016 & $0.017 \pm 0.006$ & $1.4 \pm 0.5$ \\
\hline 4 & Flare stack site & N05' 29.304' E006' 08.244' & 0.016 & 0.018 & $0.017 \pm 0.005$ & $1.4 \pm 0.4$ \\
\hline 5 & NGC Station & N05' 29.216' E006. 08.132' & 0.022 & 0.020 & $0.021 \pm 0.008$ & $1.8 \pm 0.7$ \\
\hline 6 & L \& S tango Crude flow pipe & N05' 29.285' E006” 28 185' & 0.016 & 0.014 & $0.015 \pm 0.006$ & $1.3 \pm 0.5$ \\
\hline 7 & Manifold & N05' 28.185' E006 07.720' & 0.019 & 0.018 & $0.019 \pm 0.008$ & $1.6 \pm 0.7$ \\
\hline 8 & Well 12 & N05" 29.666' E006" 06.567' & 0.020 & 0.018 & $0.019 \pm 0.007$ & $1.6 \pm 0.6$ \\
\hline 9 & Well 2 & N05” $29.219^{\prime}$ E006' 08.128' & 0.018 & 0.023 & $0.020 \pm 0.010$ & $1.7 \pm 0.8$ \\
\hline \multirow[t]{2}{*}{10} & Otor-Oweh community & N05' 29.614' E006" 06.248' & 0.012 & 0.014 & $0.013 \pm 0.005$ & $1.1 \pm 0.4$ \\
\hline & \multicolumn{2}{|l|}{ MEAN FIELD LEVEL } & & & $0.018 \pm 0.007$ & 1.50 .6 \\
\hline
\end{tabular}

Table 8: Oweh Oil and Gas Field Background Radiation Level.

\begin{tabular}{|c|c|c|c|c|c|c|}
\hline \multirow{2}{*}{$\mathrm{S} / \mathrm{N}$} & \multirow{2}{*}{ SAMPLED AREA } & \multirow{2}{*}{ GEOGRAPHICAL LOCATION } & \multicolumn{2}{|c|}{ RADIATION LEVEL $\mathrm{mRh}^{-1}$} & \multirow{2}{*}{ AVE. RAD. VALUE $\mathrm{mRh}^{-1}$} & \multirow{2}{*}{$\begin{array}{l}\text { EQ. DOSE } \\
\text { mSvy }^{-1}\end{array}$} \\
\hline & & & RAD 50 & RAD 100 & & \\
\hline 1 & Well 14 & NO5' 27.984' E006 09.856' & 0.021 & 0.015 & $0.018 \pm 0.007$ & $1.5 \pm 0.6$ \\
\hline 2. & Well 20 & NO5' 27.441' E006' 10.470"' & 0.015 & 0.021 & $0.018 \pm 0.006$ & $1.5 \pm 0.5$ \\
\hline 3 & Flow station Gate & NO5' 27.410' E006' 10.736' & 0.015 & 0.021 & $0.015 \pm 0.007$ & $1.3 \pm 0.6$ \\
\hline 4 & L \& S Tango crude flow pipe & N05' 27.422' E006' 10.778' & 0.016 & 0.019 & $0.018 \pm 0.006$ & $1.5 \pm 0.5$ \\
\hline 5 & Gas vent (knockout drum) & N05' 27.521' E006. 10.811' & 0.026 & 0.022 & $0.024 \pm 0.010$ & $2.0 \pm 0.8$ \\
\hline 6 & Flare stock site & N05' 27.541' E006” 10 826' & 0.020 & 0.024 & $0.022 \pm 0.009$ & $1.9 \pm 0.8$ \\
\hline 7 & NGC & N05' 27.303' E006 10.781' & 0.017 & 0.020 & $0.019 \pm 0.008$ & $1.6 \pm 0.7$ \\
\hline 8 & Manifold & N05" 27.226' E006" 10.702' & 0.014 & 0.015 & $0.015 \pm 0.006$ & $1.2 \pm 0.5$ \\
\hline 9 & Field logistic base (FLB) & N05" $27.256^{\prime}$ E006' 10.985' & 0.008 & 0.610 & $0.009 \pm 0.002$ & $0.8 \pm 0.2$ \\
\hline \multirow[t]{2}{*}{10} & Olomoro Community & N05' 26.989' E006" 11.820' & 0.017 & 0.018 & $0.018 \pm 0.005$ & $1.5 \pm 0.4$ \\
\hline & MEAN FIELD LEVEL & & & & $0.018 \pm 0.007$ & $1.5 \pm 0.6$ \\
\hline
\end{tabular}

Table 9: Olomoro-Oleh Oil and Gas Field Background Radiation Level.

\begin{tabular}{|c|c|c|c|c|c|c|}
\hline \multirow{2}{*}{$\mathbf{S} / \mathbf{N}$} & \multirow{2}{*}{ SAMPLED AREA } & \multirow{2}{*}{ GEOGRAPHICAL LOCATION } & \multicolumn{2}{|c|}{ RADIATION LEVEL mRh-1 } & \multirow{2}{*}{ AVE. RAD VALUE $\mathrm{mRh}^{-1}$} & \multirow{2}{*}{ EQ. DOSE mSvy ${ }^{-1}$} \\
\hline & & & RAD 50 & RAD 100 & & \\
\hline 1 & Manifold & NO5' 20.080' E006 14.865' & 0.016 & 0.015 & $0.016 \pm 0.006$ & $1.3 \pm 0.5$ \\
\hline 2. & Buster station & NO5' 20.162' E006' 14 .781"' & 0.017 & 0.014 & $0.016 \pm 0.005$ & $1.3 \pm 0.4$ \\
\hline 3 & NGC Station & NO5' 19.751' E006' 14.762' & 0.016 & 0.019 & $0.018 \pm 0.006$ & $1.5 \pm 0.5$ \\
\hline 4 & Flow station Gate & N05' 19.627' E006' 14.655' & 0.027 & 0.028 & $0.028 \pm 0.013$ & $2.3 \pm 1.1$ \\
\hline 5 & L \& S Tango crude flow pipe & N05' 19.167' E006. 14.642' & 0.022 & 0.024 & $0.23 \pm 0.010$ & $1.9 \pm 0.8$ \\
\hline 6 & Flare knock out down & N05' 19.601' E006" 14. 633' & 0.020 & 0.018 & $0.019 \pm 0.008$ & $1.6 \pm 0.7$ \\
\hline 7 & Flare stack site & N05' 19.584' E006' 14.566' & 0.017 & 0.021 & $0.019 \pm 0.007$ & $1.6 \pm 0.6$ \\
\hline 8 & Well 6 & N05" 19.251' E006" 15.960' & 0.019 & 0.023 & $0.020 \pm 0.009$ & $1.7 \pm 0.8$ \\
\hline 9 & Well 2 & N05” 19.421 ' E006' 15.862' & 0.022 & 0.026 & $0.024 \pm 0.012$ & $2.0 \pm 1.0$ \\
\hline \multirow[t]{2}{*}{10} & Uzere community & N05' 20.268' E006" 14.338' & 0.016 & 0.019 & $0.018 \pm 0.007$ & $1.5 \pm 0.6$ \\
\hline & \multicolumn{2}{|l|}{ MEAN FIELD LEVEL } & & & $0.020 \pm 0.008$ & $1.7 \pm 0.7$ \\
\hline
\end{tabular}

Table 10: Uzere East and West Oil and Gas Field Background Radiation Level. 
Citation: Avwiri GO, Agbalagba EO (2012) Studies on the Radiological Impact of Oil and Gas Activities in Oil Mineral Lease 30 (Oml30) Oil Fields in Delta State, Nigeria. J Pet Environ Biotechnol 3:115. doi:10.4172/2157-7463.1000115

Page 6 of 8

\begin{tabular}{|c|c|c|c|c|c|}
\hline Area Code & Oil and Gas Field & Host Community & Mean field dose rate $\left(\mathrm{mSvy}^{-1}\right)$ & Host Community dose rate $\left(\mathrm{mSvy}^{-1}\right)$ & Difference (\%) \\
\hline OUT & Otorugu & Otujeremi & $1.8 \pm 0.7$ & $1.8 \pm 0.6$ & 1.51 \\
\hline UEA & Ughelli West & Ekpamre & $1.6 \pm 0.4$ & $1.6 \pm 0.7$ & 0.30 \\
\hline UER & Ughelli East & Eruemukaharie & $1.5 \pm 0.6$ & $1.1 \pm 0.3$ & 39.10 \\
\hline AEM & Afiesere & Emergha & $1.7 \pm 0.7$ & $1.6 \pm 0.5$ & 7.52 \\
\hline KER & Kokori & Erhioke & $1.5 \pm 0.6$ & $1.2 \pm 0.3$ & 36.07 \\
\hline EGA & Eriemu & Gana-Agbarha & $1.4 \pm 0.6$ & $1.3 \pm 0.6$ & 6.06 \\
\hline EVN & Evwreni & Evwreni & $1.4 \pm 0.5$ & $1.3 \pm 0.5$ & 22.70 \\
\hline OWT & Oweh & Otoweh & $1.5 \pm 0.6$ & $1.1 \pm 0.4$ & 37.14 \\
\hline OLO & Olomoro-Oleh & Olomoro & $1.5 \pm 0.6$ & $1.5 \pm 0.4$ & 1.29 \\
\hline UZE & Uzere West \& East & Uzere & $1.7 \pm 0.7$ & $1.5 \pm 0.6$ & 14.4 \\
\hline
\end{tabular}

Table 11: Comparison of Studies fields and Host Communities Radiation Data.

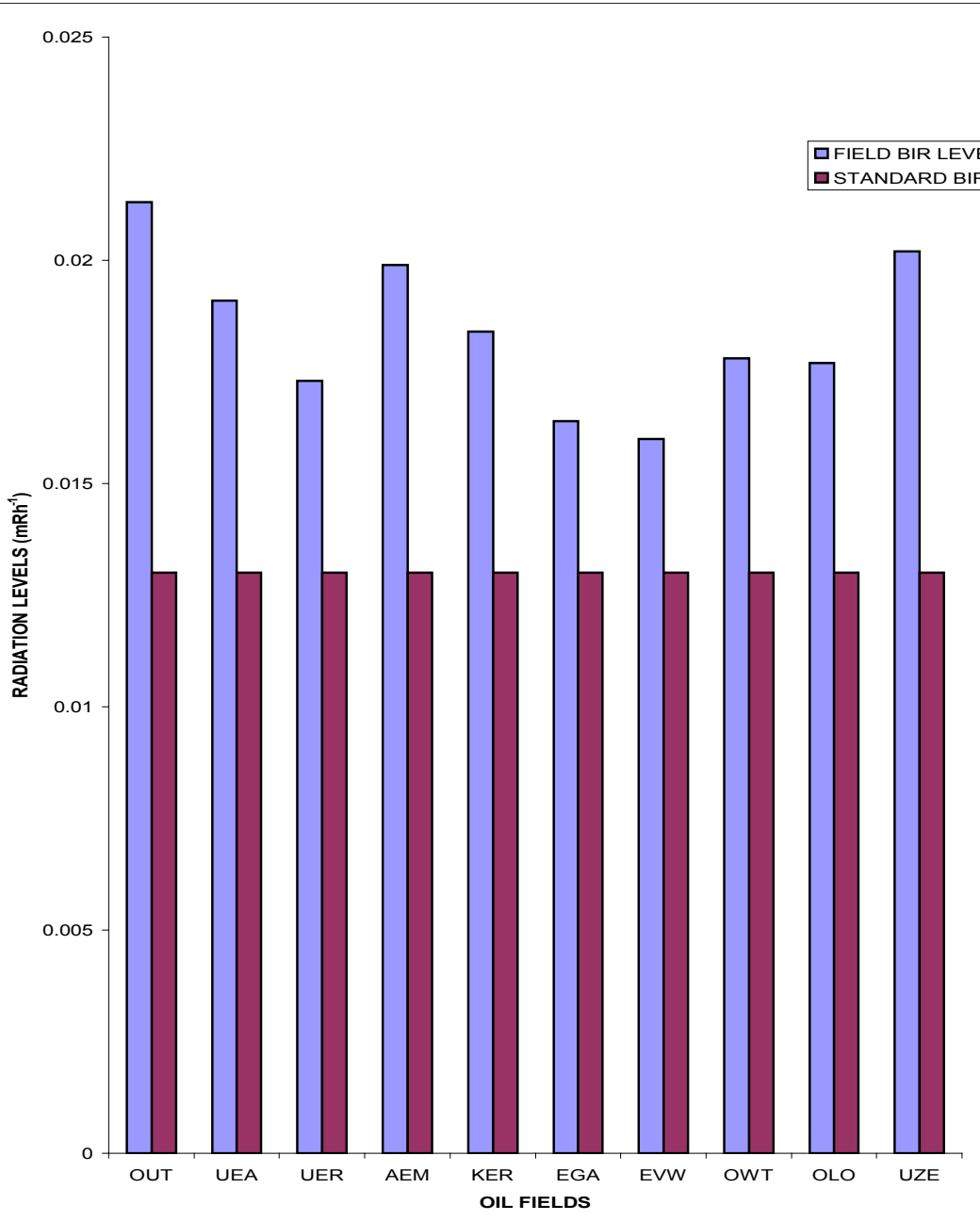

COMPARISON OF OIL FIELDS BIR LEVELS

Figure 2: Comparison of Oil fields BIR levels with Standard BIR levels.

Figure 2 shows the comparison of the mean oil and gas fields' radiation levels with the standard background radiation level of 0.013 $\mathrm{mRh}^{-1}$ recommended by ICRP [1]. The result shows that all the oil field examined exceeded the normal standard BIR level, with the maximum field exposure rate exceeding by $63.8 \%$ while the minimum mean exposure field level exceeding by $23.1 \%$. These values obtain are well above previously reported values in similar environment $[13,4,5]$.
Figure 3 shows the comparison of the host communities' average BIR levels with the normal/ standard background level of $0.013 \mathrm{mRh}^{-1}$ [1]. The results revealed that $70 \%$ of the host communities exposure rate exceeded the normal standard background radiation level, with the most impacted community (Otujeremi) being $161.5 \%$ of the standard background level while the least impact host community (Evwreni) being $84.6 \%$ of the standard background level. The control site (Non-oil bearing community) exposure rate is $69.2 \%$ of the standard background 


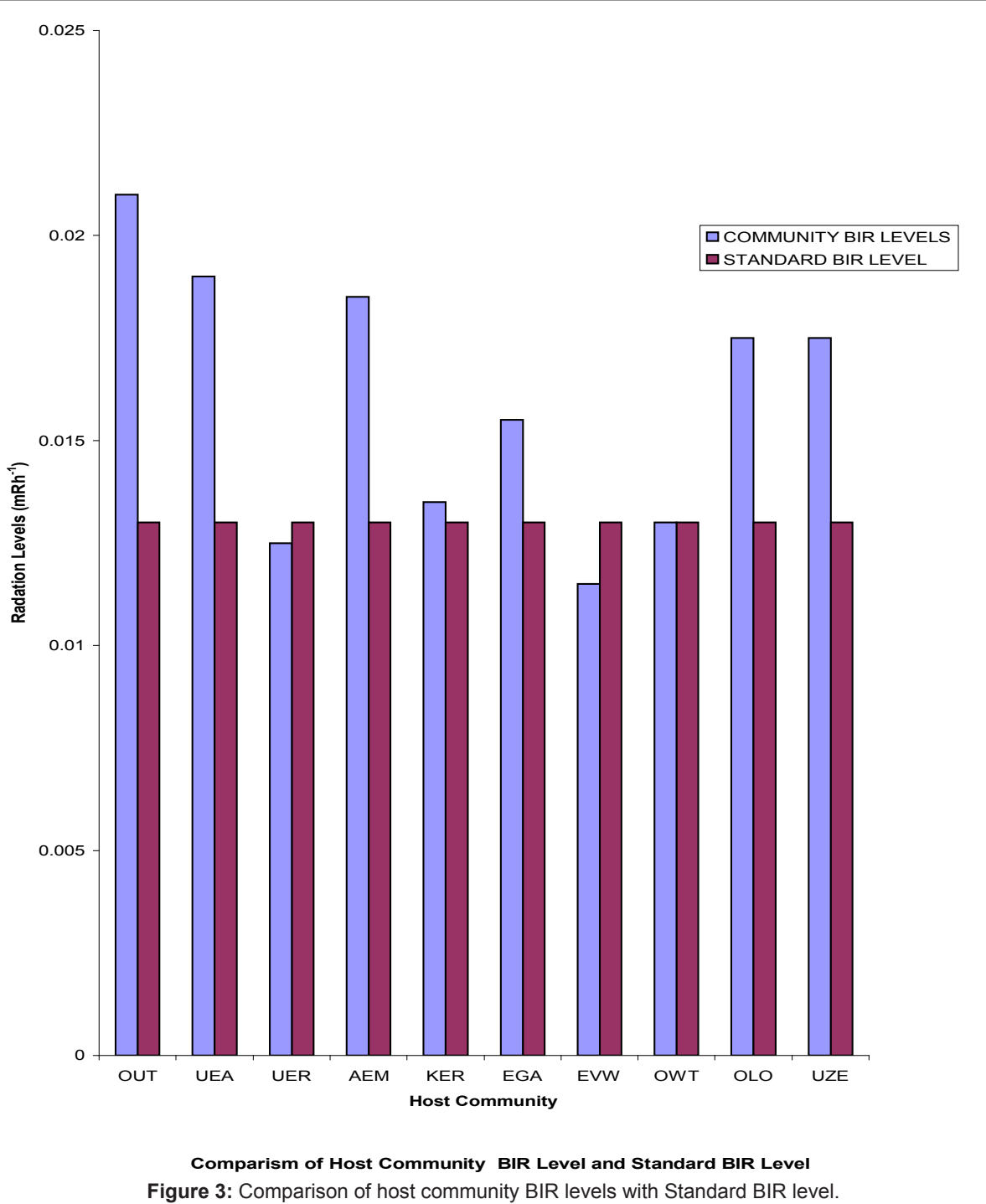

level. Examination of these three categories show that the oil bearing host communities have been impact radioactively by the operations of the oil and gas industry and their facilities in these environment.

Equivalent dose rate is the measure of the amount of radio nuclides absorbed by the human body for a given period. To avoid any somatic, epidemiological and radiological health side effect, ICRP [1] recommended and consequently set the maximum permissible limit for non- radionuclide industrial worker and the public as $1.0 \mathrm{mSvy}^{-1}$. Figure 4 shows the comparison of the mean fields' equivalent dose rate, host community dose rate and ICRP [1] maximum permissible limit. The results revealed that the dose levels in all of the fields and host communities examined exceed the maximum permissible limit for the public.

\section{Conclusion}

The investigation on the radiological impact of oil and gas activities on field workers and host communities residents, in production land area of Delta state have been conducted. The overall results obtained, show that the host communities and the field workers may have been impact negatively with radioactive elements that may be due to the oil activities in the environment. These reported values indicate no immediate side effects, but may cause long-term health hazard to both the oil field workers and residents of the host communities according to EPA [8].

Since radiation exposure in these environment may constitutes health hazard on the long term, especially to personnel and host communities. Contaminated facilities, radionuclides input materials and waste materials challenge must therefore be adequately recognize and addressed in the oil and gas industries.

We therefore, recommended as follows:

The oil and gas operating companies in these areas should put in place means of reducing their radionuclide input.

- Both life and health insurance policies should be acquired for employees and contract staff working within the flow stations, to take care of their long-term health problems.

Communities within oil and gas installation areas should have good, cheap and regular access to medical care.

- Communities within 500 meters proximity to flow stations and 


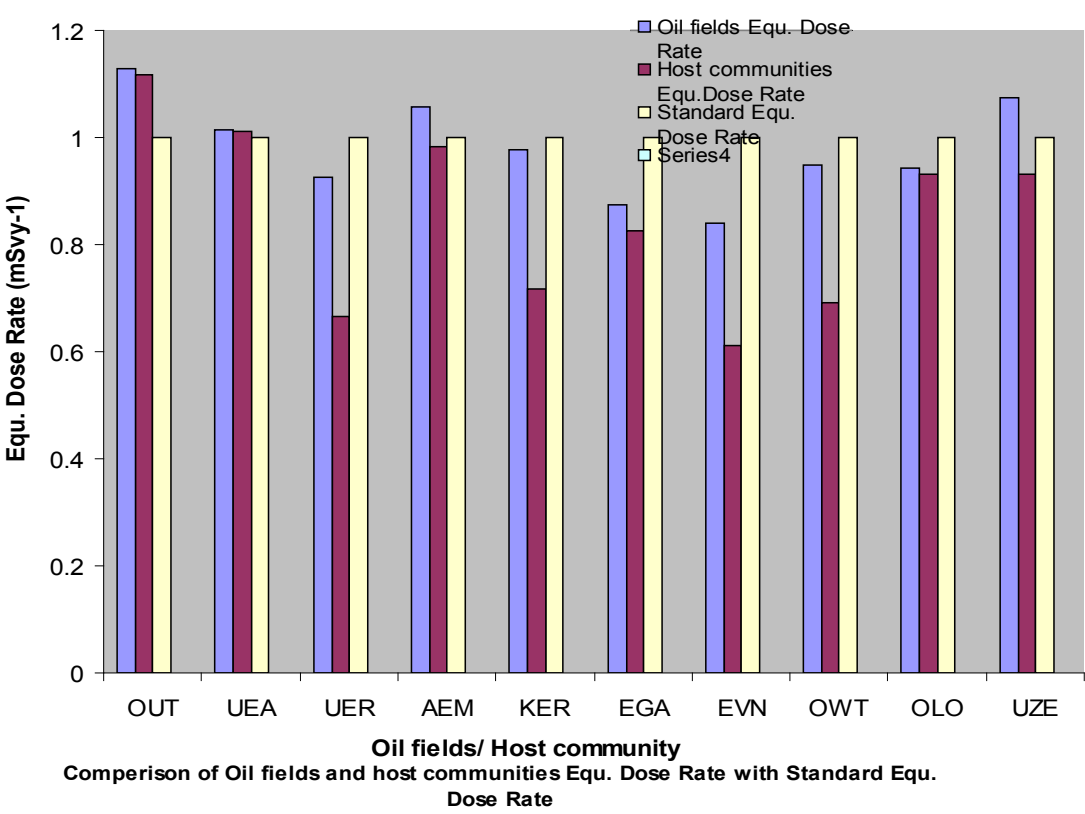

Figure 4: Comparison of Oil fields and host community Equivalent dose rates with standard Equivalent dose rate.

other oil facilities should be relocated for the safety of their health.

- All oil and gas installations should meet all known international and ISO standard.

- There should be a regular monitoring of radiation levels in these environments.

- All government agencies responsible for the safety of the environment should enforce all the existing legislation on environment protection.

\section{References}

1. International Commission on Radiological Protection (ICRP) (1999) The 1995 99 recommendation of the International Commission on Radiological Protection Publication 76. Pergamon Press.

2. Ademola JA (2008) Determination of natural radionuclide's content in some building materials in Nigeria by gamma-ray spectrometry. Health Phys 94: 4348.

3. El-Bahi SM (2004) Assessment of radioactivity and radon exhalation rate in Egyptian cements. Health Phys 86: 517-522.

4. Laogun AA, Ajayi NO, Agaja SA (2006) Variation in wellhead gamma radiation levels at the Nigeria Petroleum development company oil field, Ologbo Ede State, Nigeria. Nig J Phys 18: 135-140.

5. Avwiri GO, Agbalagba EO, Enyinna PI (2007) Terrestrial radiation around oil and gas facilities in Ughelli Nigeria. Journal of Applied Science 7: 1543-1546.

6. Elena B, Gracea C (2004) Radiological impart assessment on behalf of oil/gas industry. The Journal of Preventive Medicine 12: 16-21.

7. Rail Road Commission of Texas (RRC) 2007.

8. EPA (2009) Radiation Protection, EPA's unique role.

9. Ajayi TR, Torto N, Tchokossa P, Akinlua A (2009) Natural Radioactivity and Trace Metals in Crude oils: Implication for Health. Environ Geochem Health 31: 61-69.

10. US-EPA (United States Environmental Protection Agency) (2002) Current drinking water Standard. Office of groundwater and drinking water quality Government printing office, Washington DC, USA

11. Akpabio LE, Etuk ES, Essian K (2005) Environmental radioactive levels in Ikot Ekpen Nigeria. Nig J Space Res1: $80-87$.

12. European Council for Nuclear Research (ECNR) (1995) Safety guide for experiments at European Council for Nuclear Research, ECNR, Part III- Advice 40 , ionizing radiation

13. Arogunjo MA, Farai IP, Fuwape IA (2004) Impact of oil and gas industry to the natural radioactivity distribution in the delta region of Nigeria. Nig J Phys 16 : 131-136.

14. National Council on Radiation Protection and Measurements (NCRP) (1993) Limitation of Exposure to ionizing radiation: NCRP Report No. 116. 